Conclusions eCHP-1 represents a easy accessible protein for diagnosis and targeting in very aggressive canncers.

Disclosure Information L. Seclì: None. F. Fusella: None. M. Brancaccio: None.

\section{P03.26 IMMUNOPROFILING OF ORAL AND OROPHARYNGEAL TUMORS OF DIFFERENT ETIOLOGY}

${ }^{1}$ B Pokrýuková*, ${ }^{2} \mathrm{M}$ Grega, ${ }^{3} \mathrm{~J}$ Klozar, ${ }^{4} \mathrm{O}$ Vencalek, ${ }^{1} \mathrm{~J}$ Nunvar, ${ }^{1} \mathrm{R}$ Tachezy. ${ }^{1}$ Charles University, Faculty of Science, BIOCEV, Vestec, Czech Republic; ${ }^{2}$ Charles University, Department of Pathology and Molecular Medicine, 2nd Faculty of Medicine, Prague, Czech Republic; ${ }^{3}$ Charles University, University Hospital Motol, Department of Otorhinolaryngology and Head and Neck Surgery, 1st Faculty of Medicine, Prague, Czech Republic; ${ }^{4}$ Faculty of Science of the Palacky University in Olomouc, Department of Mathematical Analysis and Applications of Mathematics, Olomouc, Czech Republic

\subsection{6/jitc-2020-ITOC7.64}

Background Head and neck carcinomas (HNC) are the world's sixth most common cancer. Most of HNCs are associated with tobacco and other environmental factors but a growing part of oropharyngeal tumors are caused by persistent infection of human papillomavirus (HPV). Patients with HPV positive cancers have a better prognosis with fewer recurrences. This may be caused by different anti-tumor immune response and immune profile of patients. Multispectral fluorescent immunohistochemistry (fIHC) is a powerful tool for a detailed analysis of the tumor microenvironment. This method allows to access the phenotype and calculate cells in tumor parenchyma and stroma of the tumor since in comparison to flow cytometry, an architecture of the tissue remains preserved. fIHC is uniquely suited to study interaction of immune and cancer cells in situ.

Materials and Methods Number of 97 formalin fixed paraffine-embedded slides of the human HNC tissue with known etiology were examined using 4 different panels of 5 antibodies each. These panels include antibodies suitable for phenotyping of immune cells (CD3e, CD4, CD8, FOXP3) or their functional description (PD1, CTLA4, ICOS, CCR4). Additionally, antibodies against Ki67, VEGF and cell cytokeratin were used. Slides were stained using Opal ${ }^{\mathrm{TM}}$ 7-Color Fluorescent IHC Kit (Akoya Biosciences). The quantity of immune cells was evaluated in stroma and tumor compartment using InForm $^{\mathrm{TM}}$ 2.4.6. software (Akoya Biosciences). For all patients the demographic and clinical data were available and these patients were followed for up to 18 years.

Results Our results have shown significantly higher abundance of Th and Tc in both compartments of HPV+ samples. Besides HPV etiology Th and Tc in the tumor microenvironment predict independently better survival of patients. We did not observed difference in number of Tregs (characterized as a CD3+CD4+FOXP3 + cells) in tumors of different etiology, but we detected higher number of ICOS+Tregs in stroma of HPV- tumors. We also quantified the subpopulations of Th and Tc cells expressing regulatory receptors PD1 and CTLA4. PD1 showed significantly higher expression on $\mathrm{Th}$ and $\mathrm{Tc}$ both in tumor and stroma of HPV+ tumors, but CTLA4 expression was significantly higher only on Th located in stroma of HPV- tumors. Moreover, we detected significantly higher VEGF expression in both compartments and higher proliferating activity of tumor cells in HPV- tumors.

Conclusions Detailed analyses of the tumor infiltrating lymphocytes allows for selection of prognostic markers in HNC of different etiology. Our results may also help to understand the better prognosis of HPV + patients. More detailed survival analyses with inclusion of other clinical and demographic data will be presented.

Disclosure Information B. Pokrývková: None. M. Grega: None. J. Klozar: None. O. Vencalek: None. J. Nunvar: None. R. Tachezy: None.

\section{P03.27 ROLE OF NOX2 FOR HYPOXIA-INDUCED CHEMORESISTANCE IN ACUTE MYELOID LEUKEMIA}

S Paul*, H Grauers Wiktorin, R Kiffin, K Hellstrand, A Martner. TIMM Laboratory, Sahlgrenska Cancer Center, Institute of Biomedicine, Sahlgrenska Academy, University of Gothenburg, Gothenburg, Sweden

\subsection{6/jitc-2020-ITOC7.65}

Background Relapse of acute myeloid leukemia (AML) may arise from residual chemoresistant leukemic cells. A hypoxic tumor microenvironment, such as the bone marrow, is known to enhance chemoresistance in various forms of cancer, including AML. Hypoxia inducible factor 1 alpha (HIF-1 $\alpha)$ is an important mediator of cellular adaptation to hypoxia. HIF- $1 \alpha$ is a constitutively expressed transcription factor that is rapidly degraded under normoxic conditions after hydroxylation by oxygen sensors, such as the HIF prolyl hydroxylases (PHDs). However, under hypoxic conditions the oxygen sensors lose the ability to induce the degradation of HIF- $1 \alpha$ resulting in its stabilization and translocation to the nucleolus where it induces the transcription of genes associated with glucose metabolism, angiogenesis, and cell survival. This may result in proliferation of malignant cells, impaired tumor cell differentiation and chemoresistance. Reactive oxygen species (ROS) have been shown to inhibit PHDs and may thereby stabilize HIF-1 $\alpha$, and may thus contribute to chemoresistance. AML cells may generate ROS via the myeloid NADPH oxidase NOX2. We therefore hypothesized that NOX inhibitors would decrease chemoresistance in a hypoxic environment.

Materials and Methods The wild type (WT) AML cell line PLB-985 and its NOX2 knocked out (KO) counterpart were cultured for five days in hypoxia ( $1 \%$ oxygen) or normoxia (21\% oxygen) in the presence or absence of the NOX inhibitors histamine dihydrochloride (HDC), diphenyleneiodonium (DPI) and GSK2795039. Thereafter cells were exposed to the chemotherapeutic agent daunorubicin for 48 hours (in hypoxia or normoxia) and cell death was determined using the XTT assay. Stabilization of HIF- $1 \alpha$ was measured either by western blot or flow cytometry. Differentiation of cells was quantified by measuring the expression of CD14 and CD11b by flow cytometry.

Results Hypoxia reduced the sensitivity of WT PLB-985 cells to daunorubicin induced cell death $(P<0.05, \mathrm{n}=4)$ whereas NOX2 KO cells were equally sensitive to daunorubicin in hypoxia and normoxia $(P>0.5, \mathrm{n}=4)$. Furthermore, NOX2 KO AML cells displayed increased sensitivity to daunorubicin induced killing compared with PLB WT cells in a hypoxic environment $(P<0.05, \mathrm{n}=4)$. Preliminary results show that pharmacological NOX inhibition using DPI enhanced the sensitivity of WT AML cells to daunorubicin induced killing. These results suggests that functional NOX2 contributes to chemoresistance in a hypoxic environment. As expected, hypoxia stabilized the expression of HIF- $1 \alpha$ in AML cells. Preliminary results suggest that HIF- $1 \alpha$ expression was reduced in the presence of NOX inhibitors. 
Conclusions Genetic deletion or pharmacological inhibition of NOX2 sensitized AML cells to daunorubicin induced killing in hypoxic environments. NOX2 may thus be a target for overcoming chemoresistance in AML cells in the hypoxic bone marrow environment.

Support This work was supported by Assar Gabrielsson's Foundation( FB19-64) and other grants used by the research group.

Disclosure Information S. Paul: None. H. Grauers Wiktorin: E. Ownership Interest (stock, stock options, patent or other intellectual property); Modest; Patent. R. Kiffin: None. K. Hellstrand: E. Ownership Interest (stock, stock options, patent or other intellectual property); Modest; Patent. A. Martner: E. Ownership Interest (stock, stock options, patent or other intellectual property); Modest; Patent.

\section{P03.28 STRUCTURAL CHARACTERISTICS IN TUMOR AND LYMPH NODES AS PREDICTORS OF 3-YEAR METASTASIS-FREE SURVIVAL IN SURGICALLY TREATED NSCLC}

${ }^{1} \mathrm{~L}$ Sellmer* ${ }^{2}{ }^{2} \mathrm{~J}$ Kovacs, ${ }^{3} \mathrm{~J}$ Neumann, ${ }^{1} \mathrm{R}$ Kiefl, ${ }^{1} \mathrm{D}$ Kauffmann-Guerrero, ${ }^{2} \mathrm{C}$ Schneider, ${ }^{1}$ A Tufman. ${ }^{1}$ Department of Thoracic Oncology, Hospital of the Ludwig-Maximilians University, Munich, Germany; ${ }^{2}$ Department of Thoracic Surgery, Hospital of the LudwigMaximilians University, Munich, Germany; ${ }^{3}$ Department of Pathology, Ludwig-Maximilians University, Munich, Germany

\subsection{6/jitc-2020-ITOC7.66}

Background Surgery is the treatment of choice for early and for some locally advanced non-small cell lung cancer (NSCLC). Ipsilateral hilar and mediastinal lymph nodes are generally removed at the time of tumor resection and assessed for tumor infiltration. However, in particular in the context of immunotherapy, there is now increased awareness about the physiological role of lymph nodes in cancer. It may be possible to assess immune response by examining the cellular composition of locoregional lymph nodes. We aimed to assess structural characteristics in tumor tissue and affected and unaffected lymph nodes in patients with and without 3-year metastasis-free survival.

Materials and methods Internal hospital databases were screened for NSCLC patients fulfilling inclusion criteria. Data on patients age, sex, surgery type, (neo)adjuvant therapy, tumor characteristics and time and location of relapse was extracted. FFPE tissue blocks of primary tumor, affected and unaffected lymph nodes were collected. Hematoxylin and eosin stainings were obtained and tissues were analyzed (e.g. for B-cell proliferation and macrophage infiltration) in collaboration with an experienced pathologist.

Results A total of 754 NSCLC patients were screened for inclusion criteria. Of these, 71 patients remained in remission for at least 3 years after surgery, and 80 patients had local or systemic relapse within 3 years after surgery. Structural characteristics in tumor and lymph node immune populations differed between patients with and without 3-year metastasis-free survival.

Conclusion Structural characteristics differ between patients with and without relapse. Our findings show that structural markers in tumor and lymph nodes should be taken into account when assessing patient prognosis and relapse risk.

Disclosure Information L. Sellmer: None. J. Kovacs: None. J. Neumann: None. R. Kiefl: None. D. Kauffmann-Guerrero: None. C. Schneider: None. A. Tufman: None.

\section{P03.29 CHARACTERIZATION OF TREATMENT-INDUCED ADAPTIVE IMMUNE RESPONSES IN PANCREATIC DUCTAL ADENOCARCINOMA}

${ }^{1,2}$ J Heetmeyer ${ }^{*},{ }^{1,2} \mathrm{C}$ Falcomatà, ${ }^{1,2} \mathrm{~S}$ Bärthel, ${ }^{2,3} \mathrm{C}$ Schneeweis, ${ }^{1,2} \mathrm{~A}$ Coluccio,

${ }^{1,2} \mathrm{C}$ Veltkamp, ${ }^{2,3} \mathrm{G}$ Schneider, ${ }^{1,2} \mathrm{D}$ Saur. ${ }^{1}$ Institute of Translational Cancer Research and Experimental Cancer Therapy, TranslaTUM, Technical University Munich, München, Germany; ${ }^{2}$ German Cancer Consortium (DKTK), German Cancer Research Center (DKFZ), Heidelberg, Germany; ${ }^{3}$ Department of Internal Medicine II, Klinikum rechts der Isar, Technical University Munich, München, Germany

\subsection{6/jitc-2020-ITOC7.67}

Background Pancreatic ductal adenocarcinoma (PDAC) is a highly aggressive malignancy marked by poor prognosis and profound drug resistance characterized in more than $90 \%$ of cases by KRAS mutations. To recapitulate central aspects of PDAC, we employed genetically engineered mouse models presenting Kras ${ }^{\mathrm{G} 12 \mathrm{D}}$ pancreas specific expression. Through a high-throughput combination drug screen with trametinib as backbone we identified a high synergism with the multikinase inhibitor nintedanib, preferentially in mesenchymal PDAC, a subtype of this disease characterized by poor prognosis and therapeutic resistance. This combinatorial treatment, that led to the induction of apoptosis in vitro and disease regression in vivo, was accompanied by a strong tumor infiltration of CD8 positive T cells.

Materials and Methods To characterize the treatment-induced adaptive immune cell infiltration in vivo, we performed orthotopic transplantations of KRAS-driven murine PDAC cell lines presenting mesenchymal and epithelial morphology. The derived control and nintedanib + trametinib treated PDAC tumors were analyzed by multi-color immunofluorescence stainings. We compared the findings to high parameter flow cytometry results.

Results Confocal microscopy of the immunofluorescence stainings revealed an overall increase of tumor-infiltrating lymphocytes (TIL) in the tumors upon combinatorial treatment with substantial differences in quantity and spatial distribution. Tumors derived from a PDAC cell line of epithelial morphology were characterized by few TIL mainly located at the invasive margins of the tumors, while tumors derived from a mesenchymal PDAC cell line showed a strong increase of TIL even in the center of the tumor mass. Furthermore, an increased ratio of CD8 positive cytotoxic T cells to CD4 positive helper $\mathrm{T}$ cells as well as a decrease of Foxp3 and CD4 positive regulatory $\mathrm{T}$ cells could be observed for tumors derived from the mesenchymal PDAC cell line under combinatorial treatment. To investigate if the observed recruitment of $\mathrm{T}$ cells was indispensable for treatment efficacy of the combinatorial therapy, we orthotopically transplanted the mesenchymal PDAC cell line in immunodeficient CD3-Knockout (CD3ko) mice and applied an analogous combinatorial treatment scheme. In the CD3ko mice, the combinatorial treatment did not lead to an increased survival or tumor regression as observed in immunocompetent mice. However, flow cytometry and immunofluorescence stainings revealed an increase of $\mathrm{B}$ cells upon nintedanib + trametinib treatment.

Conclusions Our findings indicate a reduced efficacy of the combinatorial treatment in $\mathrm{T}$ cell deficient mice, underlining the importance of $\mathrm{T}$ cells in treatment-induced anti-tumor responses and enlarging the understanding of the role of TIL in PDAC.

Disclosure Information J. Heetmeyer: None. C. Falcomatà: None. S. Bärthel: None. C. Schneeweis: None. A. Coluccio: None. C. Veltkamp: None. G. Schneider: None. D. Saur: None. 